\title{
Unique factorization in perturbative QFT
}

\author{
D. Kreimer ${ }^{\text {** }}$ \\ ${ }^{\mathrm{a}}$ CNRS-IHES \\ 35 rte. de Chartres, F-91440 Bures-sur-Yvette, France
}

We discuss factorization of the Dyson-Schwinger equations using the Lie- and Hopf algebra of graphs. The structure of those equations allows to introduce a commutative associative product on 1PI graphs. In scalar field theories, this product vanishes if and only if one of the factors vanishes. Gauge theories are more subtle: integrality relates to gauge symmetries.

\section{The Lie and Hopf algebra of graphs}

Over recent years, the Lie and Hopf algebra structures of Feynman graphs have been firmly established in collaboration with Alain Connes and David Broadhurst [1-11]. Hopefully, they find their way now into the algorithmic toolkit of the practitioner of QFT. They directly address the computational practice of momentum space Feynman integrals, but can be equivalently formulated in coordinate space. They take into account faithfully the self-similarity of physics at different scales and the iteration of Green functions in terms of itself by their quantum equations of motions, the Dyson-Schwinger equations. We will review quickly these Lie and Hopf algebra structures and then report in the next section on factorization properties which exist in the formal series over graphs contributing to a given Green function. We motivate this factorization in comparison with the factorization of integers leading to an Euler product for $\zeta$-functions, and comment on the fate of such factorizations in gauge theories. Results reported here can also be found in [12], which in particular contains a more extended discussion of factorization properties and their implications on perturbative expansions with regard to the question if the factorization commutes with the application of the Feynman rules. We start our summary with the pre-Lie structure.

\footnotetext{
*supported in parts by NSF grant 0205977; Ctr. Math.
} Phys. at Boston U., BUCMP/02-06

\subsection{The Pre-Lie Structure}

A 1PI Feynman graph $\Gamma_{1}$ provides internal edges and vertices. Each internal vertex $v$ has a set of edges $f_{v}$ attached to it. A vertex correction graph $\Gamma_{2}$ has a set of external edges $\Gamma_{2, \text { ext }}^{[1]}$ attached to it. If those two sets agree, we can glue $\Gamma_{2}$ into $\Gamma_{1}$ at $v$, where we sum over all these possible bijections between $f_{v}$ and $\Gamma_{2 \text {,ext }}^{1}$, and normalize such that topologically different graphs are generated precisely once. Furthermore, we formally define this insertion to vanish when the two sets do not agree. Summing over all places $v$ in $\Gamma_{1}$ gives a bilinear map $*$ from $1 \mathrm{PI}$ graphs to $1 \mathrm{PI}$ graphs. It can be extended to insertions of selfenergies into edges in the obvious manner $[5,7]$. One then has:

Theorem $1[5,1,7]$ The operation $*$ is pre-Lie:

$$
\begin{aligned}
& {\left[\Gamma_{1} * \Gamma_{2}\right] * \Gamma_{3}-\Gamma_{1} *\left[\Gamma_{2} * \Gamma_{3}\right] } \\
= & {\left[\Gamma_{1} * \Gamma_{3}\right] * \Gamma_{2} \quad-\quad \Gamma_{1} *\left[\Gamma_{3} * \Gamma_{2}\right] . }
\end{aligned}
$$

The theorem says that the lack of associativity in the bilinear operation $*$ is invariant under permutation of the elements indexed 2,3. This suffices to show that the antisymmetrization of this map fulfils a Jacobi identity. Hence we get a Lie algebra $\mathcal{L}$ by antisymmetrizing this operation:

$$
\left[\Gamma_{1}, \Gamma_{2}\right]=\Gamma_{1} * \Gamma_{2}-\Gamma_{2} * \Gamma_{1},
$$

and a Hopf algebra $\mathcal{H}$ as the dual of the universal enveloping algebra of this Lie algebra $[5,4]$. We restrict attention to graphs which are superficially divergent, while superficially convergent graphs can be incorporated in a trivial manner 


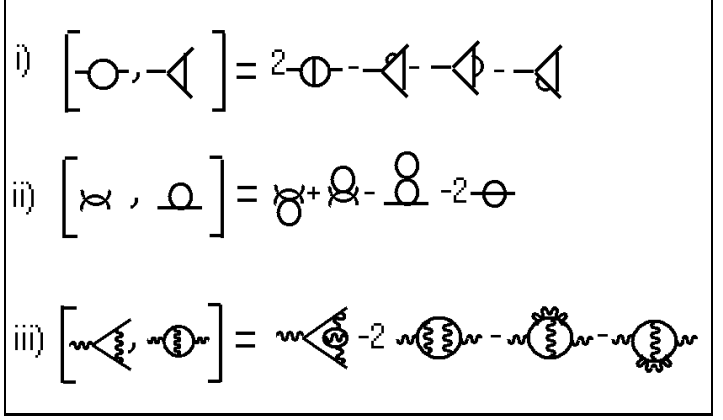

Figure 1. Examples of Lie brackets in various theories: i) $\phi_{6}^{3}$ graphs, ii) $\phi_{4}^{4}$ graphs, iii) QED graphs.

[5]. Fig.(1) gives Lie brackets for various different theories.

\subsection{The principle of multiplicative sub- traction}

The beauty of the existence of these graded combinatorial Lie algebras is that they make the role of locality manifest. We dualize the universal enveloping algebra $\mathcal{U}(\mathcal{L})$ of $\mathcal{L}$ and obtain a commutative, non-cocommutative Hopf algebra $\mathcal{H}$ [5]. To find this dual, one uses a Kronecker pairing and constructs it in accordance with the Milnor-Moore theorem $[5,7,4]$.

1PI graphs are the linear generators of the Hopf algebra, with their disjoint union furnishing a commutative product in the algebra. We then identify the Hopf algebra by a coproduct $\Delta: \mathcal{H} \rightarrow \mathcal{H} \otimes \mathcal{H}:$

$\Delta(\Gamma)=\Gamma \otimes 1+1 \otimes \Gamma+\sum_{\gamma \subset \Gamma} \gamma \otimes \Gamma / \gamma$,

the sum is over all unions of one-particle irreducible (1PI) superficially divergent proper subgraphs. We extend this definition to products of graphs so that we get a bialgebra. The above sum should, when needed, also run over appropriate projections to formfactors, to specify the appropriate type of local insertion [5] which appear in local counterterms, which we omitted in the above sum for simplicity. Fig.(2) gives exam-

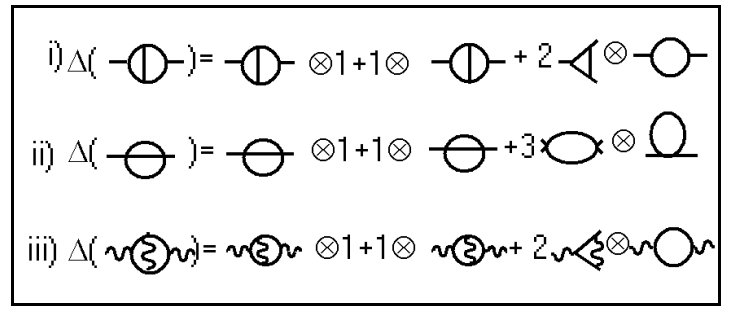

Figure 2. Examples for coproducts $\Delta(\Gamma)$ in various theories: i) $\phi_{6}^{3}$, ii) $\phi_{4}^{4}$, iii) QED.

ples.

We still need a counit and antipode (coinverse): the counit $\bar{e}$ vanishes on any non-trivial Hopf algebra element, $\bar{e}(1)=1, \bar{e}(X)=0$. At this stage we have a commutative, but typically not cocommutative bialgebra. It actually is a Hopf algebra as the antipode (coinverse) in such circumstances comes for free as

$S(\Gamma)=-\Gamma-\sum_{\gamma \subset \Gamma} S(\gamma) \Gamma / \gamma$.

Those ingredients give us a group structure on any multiplicative map from the Hopf algebra to some ring or algebra $V$ : the co-unit, -product, -inverse give us a unit, product and inverse on such maps.

As physicists, we have a distinguished such map, (momentum-space) Feynman rules $\phi: \mathcal{H} \rightarrow$ $V$ from the Hopf algebra of graphs $\mathcal{H}$ into an appropriate space $V$, of, say, Laurent polynomials in the dimensional regularization parameter $\varepsilon$.

We will have to make one further choice: a renormalization scheme. For us, this is a map $R: V \rightarrow V$ faithful on short distance singularities, which furthermore obeys

$R(x y)+R(x) R(y)=R(R(x) y)+R(x R(y))$,

an equation which guarantees the multiplicativity of renormalization and is at the heart of the Birkhoff decomposition of $[5,6]$.

Renormalization theory emerges as a principle of multiplicative subtraction: we define a further character $S_{R}^{\phi}$ which deforms $\phi \circ S$ slightly and 
delivers the counterterm for $\Gamma$ in the renormalization scheme $R$ :

$S_{R}^{\phi}(\Gamma)=-R[\phi(\Gamma)]-R\left[\sum_{\gamma \subset \Gamma} S_{R}^{\phi}(\gamma) \phi(\Gamma / \gamma)\right]$

which should be compared with the undeformed

$\phi \circ S=-\phi(\Gamma)-\sum_{\gamma \subset \Gamma} \phi \circ S(\gamma) \phi(\Gamma / \gamma)$.

We obtain the renormalization of $\Gamma$ by the application of a renormalized character

$$
\Gamma \rightarrow S_{R}^{\phi} \star \phi(\Gamma)
$$

and Bogoliubov's $\bar{R}$ operation as

$$
\bar{R}(\Gamma)=\phi(\Gamma)+\sum_{\gamma \subset \Gamma} S_{R}^{\phi}(\gamma) \phi(\Gamma / \gamma),
$$

so that we have

$S_{R}^{\phi} \star \phi(\Gamma)=\bar{R}(\Gamma)+S_{R}^{\phi}(\Gamma)$.

Here, $S_{R}^{\phi} \star \phi$ is an element in the group of characters of the Hopf algebra, with the previously announced group law given by

$$
\phi_{1} \star \phi_{2}=m_{V} \circ\left(\phi_{1} \otimes \phi_{2}\right) \circ \Delta,
$$

so that indeed the coproduct, counit and coinverse (the antipode) give the product, unit and inverse of this group, as it befits a Hopf algebra. This Lie group has the previous Lie algebra $\mathcal{L}$ of graph insertions as its Lie algebra [5].

\section{Factorization}

There is a connection between Dyson-Schwinger equations and Euler products [12]. To motivate it let us start with the Riemann $\zeta$ function, and obtain it as a solution to a DysonSchwinger equation, as in [12]. This is only meant as a sufficient stimulus to invert the reasoning and look for Euler products in quantum field theory. This whole section is meant to inspire an investigation of the question how such factorizations of Dyson-Schwinger equations actually fare when we apply Feynman rules or related characters to Euler products, a question addressing the transition from the perturbative to the nonperturbative [12]. It will be discussed in detail in upcoming work of the author.

\subsection{The Riemann $\zeta$-function from a Dy- son-Schwinger equation}

The Riemann $\zeta$-function is the analytic continuation of the sum $\sum_{n} 1 / n^{s}$, and has an Euler product

$\zeta(s)=\sum_{n} \frac{1}{n^{s}}=\prod_{p} \frac{1}{1-p^{-s}}, \Re(s)>1$,

over all primes $p$ of the (rational) integers.

There is a well-known Hopf algebra of sequences $\left(p_{1}, \ldots, p_{k}\right)$ (the $p_{i}$ are primes), and we can introduce $B_{+}^{p}[J]$ as the sequence which is obtained by adding a new prime $p$ as the first element to the sequence $J$. The Hopf algebra structure emerges when we require that $B_{+}^{p}$ is Hochschild closed for all $p[12,4]$ :

$\Delta\left(B_{+}^{p}[J]\right)=B_{+}^{p}[J] \otimes 1+\left[\mathrm{id} \otimes B_{+}^{p}\right] \Delta[J]$,

with $\Delta(1)=1 \otimes 1$ and we identify 1 with the empty sequence. Define the value $w(J)$ to be the product of the entries of $J$, and let the symmetry factor $S(J)$ be the number of sequences which have the same value, which simply is $k$ ! if the sequence has length $l(J)=k$. Note that for a one element sequence $(p)$,

$\Delta[(p)]=(p) \otimes 1+1 \otimes(p)$,

primitive elements have prime value, $w((p))=p$.

Consider the "Dyson-Schwinger equation"

$\bar{\zeta}(\rho)=1+\rho \sum_{p} B_{+}^{p}[\bar{\zeta}(\rho)]$

We obtain a formal series (in "the coupling" $\rho$ )

$\bar{\zeta}(\rho)=1+\rho \sum_{p}(p)+\rho^{2} \sum_{p_{1}, p_{2}}\left(p_{1}, p_{2}\right)+\cdots$.

Define "Feynman rules" by $\phi_{s}(J)=\frac{1}{l(J) !} w(J)^{-s}$, and set

$\zeta(s, \rho)=\phi_{s}[\bar{\zeta}(\rho)]$.

We regain Riemann's $\zeta$ function as

$\zeta(s)=\lim _{\rho \rightarrow 1} \zeta(s, \rho)$.

Note the general structure of the formal "Dyson-Schwinger equation" above: it determines an 
unknown $\bar{\zeta}(\rho)$ in terms of itself, as "1 plus a sum over the image of the unknown $\bar{\zeta}(\rho)$ under all closed Hochschild one cocycles $B_{+}^{p}$, weighted by appropriate symmetry factors". Is there an Euler product for $\bar{\zeta}$ ?

The simplest way is to get it from the wellknown shuffle product on sequences.

$$
\begin{aligned}
B_{+}^{p_{1}}\left(J_{1}\right) \sqcup B_{+}^{p_{2}}\left(J_{2}\right) & =B_{+}^{p_{1}}\left(J_{1} \sqcup B_{+}^{p_{2}}\left(J_{2}\right)\right) \\
& +B_{+}^{p_{2}}\left(B_{+}^{p_{1}}\left(J_{1}\right) \sqcup J_{2}\right) .
\end{aligned}
$$

Then,

$\bar{\zeta}(\rho)=\Pi_{p}^{\sqcup} \frac{1}{1-\rho(p)}$,

$(1-\rho(p))^{-1}=1+\rho(p)+\rho^{2}(p) \sqcup(p)+\cdots$, and where the shuffle product is used in the Euler product throughout. We have

$\zeta(s)=\phi_{s}\left(\Pi_{p}^{\sqcup} \frac{1}{1-\rho(p)}\right)=\Pi_{p} \frac{1}{1-p^{-s}}$,

the evaluation of the product is the product of the evaluations.

The reason we dared calling the above equation a Dyson-Schwinger equation is a simple fact - the true Dyson-Schwinger equations of QFT have a similar structure: they express an unknown Green function as a sum over all possible insertions of itself in all possible skeleton diagrams. This allows to write the unknown Green function as a sum over all possible images over all closed Hochschild one-cocycles in the theory $[12,13]$, precisely provided by the primitive bidegree one graphs $\gamma$, which play the role of primes.

\subsection{Factorizing graphs}

Can a factorization into Euler products be found in quantum field theory? And if it can be found at the combinatorial level, will the evaluation, by the Feynman rules, equal the product of the evaluations, and, if not, how will it deviate?

A typical Dyson-Schwinger equation is of the form [12]

$X=1+\sum_{\gamma} B_{+}^{\gamma}\left(g^{k}\left[\cup_{k} X\right]\right)$,

where the infinite sum in the Hopf algebra is over primitive graphs $\gamma, k=k(\gamma)$ is the loop-degree

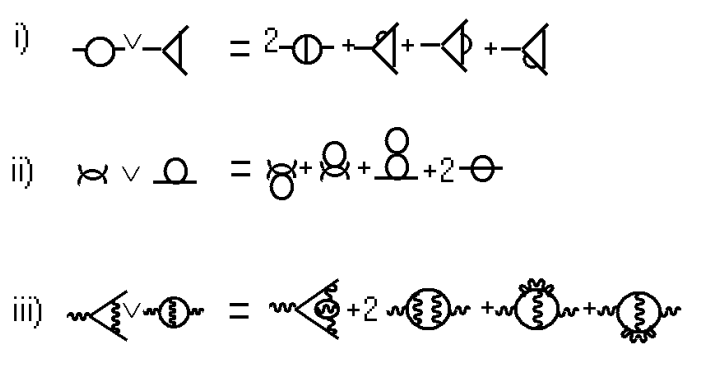

Figure 3. Examples of $\vee$-products in various theories: i) $\phi_{6}^{3}$, ii) $\phi_{4}^{4}$, iii) QED. In these simple examples, they correspond just to a symmetrization instead of an antisymmetrization of the pre-Lie insertion. The general definition is given in [12].

of $\gamma$, and as the notation indicates, the maps $B_{+}^{\gamma}$ are closed Hochschild one-cocycles, and the sum is over all of those $[12,4] . X$ is an infinite sum of graphs contributing to a chosen Green function, and evaluation by the Feunman rules delivers the usual Dyson-Schwinger equations given as an integral equation over the kernels provided by the primitive graphs $\gamma$. Note that, as insertion into a primitive graph commutes with the coproduct in the desired way, we can directly read-off the renormalized Dyson-Schwinger equation as

$X_{R}=Z_{X}+\sum_{\gamma} B_{+}^{\gamma}\left(g^{k}\left[\cup_{k} X_{R}\right]\right)$,

where the $Z$-factor $Z_{X}$ is the negative part in the Birkhoff decomposition with respect to a renormalization scheme $R$, as usual.

The first question to ask is: is there a combinatorial Euler product for the formal sum over graphs generated by such an equation? The answer is indeed affirmative.

The crucial step lies in the definition of an associative product $\vee$ which generalizes the shuffle product $\sqcup$ appropriate for totally ordered sequences to the partial order given by being a subgraph [12]. We simply describe it in Fig.(3).

Then, we have

Theorem $2 X(g)=\prod_{\gamma}^{\vee} \frac{1}{1-g^{k(\gamma)} \gamma}$, 
the proof of which is to be given elsewhere.

Here is not the space to discuss the fascinating question of how much we can say about

$$
\phi\left(\prod_{\gamma}^{\vee} \frac{1}{1-\gamma}\right) \text { vs } \prod_{\gamma} \frac{1}{1-\phi(\gamma)}=\zeta_{G}(\phi) ?
$$

A discussion of this question was started in [12], and answers are in preparation. Note that such factorization suggest pseudo-exponentiations of transcendentals in the Taylor expansion of skeleton graphs in the dimensional regularization parameter, as reported by David Broadhurst at this conference [14].

\subsection{Gauge symmetries}

Finally, let us mention a first simple example as to how basic algebraic structures relating to such factorizations in Dyson-Schwinger equations relate to physical properties of a theory. Let $\gamma_{\mathrm{vp}}$ denote the one-loop vacuum-polarization in QED, and more generally let $\left\{\Gamma_{\mathrm{N}-\mathrm{A}}\right\}$ be the set of $1 \mathrm{PI}$ graphs in a non-abelian gauge theory which have as external lines two or more gauge bosons and such that the three-valent fermion-boson vertex is the only type of vertex which appears in them. They hence all have at least one fermion loop.

Proposition 3 i) The product $\Gamma_{1} \vee \Gamma_{2}$ is integral for $1 P I$ graphs in $\phi_{6}^{3}$ and $\phi_{4}^{4}$.

ii) It is non-integral for gauge theories:

$\Gamma_{1} \vee \Gamma_{2}=0 \Rightarrow \Gamma_{1}=0$ or $\Gamma_{2}=0$ or $\Gamma_{1}=\Gamma_{2}=\gamma_{\mathrm{vp}}$ (QED);

$\Gamma_{1} \vee \Gamma_{2}=0 \Rightarrow \Gamma_{1}=0$ or $\Gamma_{2}=0$ or $\Gamma_{1}, \Gamma_{2} \in$ $\left\{\Gamma_{\mathrm{N}-\mathrm{A}}\right\}$ (non-abelian case).

Proof: i) Each 1PI graph with one or more loops contains internal edges and vertices. As these theories are renormalizable and we restrict the Hopf algebra to superficially divergent graphs, the product $\mathrm{V}$, symmetrizing over insertions of superficially divergent graphs into each other, will not vanish. ii) QED: the one-loop vacuum polarization has two internal fermion propagators and two internal vertices, but no internal photon line. But it is itself a self-energy graph for the photon. Its $\vee$-product with itself hence vanishes. Each other QED graph $\Gamma$ has internal vertices, fermion lines and photon lines, so that $\Gamma \vee \gamma=0$ implies $\gamma=0$. For the non-abelian case, this argument has a natural generalization to the indicated set of graphs, and relates to questions of uniqueness of factorization [13].

This relates integrality of the $V$-product to gauge/BRST symmetries. For example, the Hopf algebra of QED graphs can be divided by an appropriate ideal of graphs such that the quotient consists of graphs free of one-loop vacuumpolarization subgraphs, and this quotient turns out to be equivalent to the ideal generated by the Ward identities. This deserves a much more detailed discussion again to be given elsewhere.

\section{REFERENCES}

1. D. Kreimer, Adv. Theor. Math. Phys. 2 (1998) 303[q-alg/9707029].

2. D. Kreimer, Adv. Theor. Math. Phys. 3 (2000) 627[hep-th/9901099].

3. D. Kreimer, Commun. Math. Phys. 204 (1999) 669[hep-th/9810022].

4. A. Connes, D. Kreimer, Commun. Math. Phys. 199 (1998) 203[hep-th/9808042].

5. A. Connes, D. Kreimer, Commun. Math. Phys. 210 (2000) 249[hep-th/9912092].

6. A. Connes, D. Kreimer, Commun. Math. Phys. 216 (2001) 215[hep-th/0003188].

7. A. Connes, D. Kreimer, Annales Henri Poincaré 3 (2002) 411[arXiv:hep-th/0201157].

8. D. J. Broadhurst, D. Kreimer, J. Symb. Comput. 27 (1999) 581[hep-th/9810087].

9. D. J. Broadhurst, D. Kreimer, Commun. Math. Phys. 215 (2000) 217[hep-th/0001202].

10. D. J. Broadhurst, D. Kreimer, Phys. Lett. B475 (2000) 63[hep-th/9912093].

11. D. J. Broadhurst, D. Kreimer, Nucl. Phys. B600 (2001) 403[hep-th/0012146].

12. D. Kreimer, New mathematical structures in renormalizable quantum field theories, invited contribution, Annals of Physics, in press, preprint at http://www.esi.ac.at, ESI-1236.

13. D. Kreimer, Structures in Feynman graphs Hopf algebras and Symmetries, talk given at the Dennisfest, Stony Brook June 14-21, 2001 [hep-th/0202110], to appear.

14. D. J. Broadhurst, Where do the tedious products of $\zeta$ 's come from?, hep-ph/0211194. 